\title{
Brain Tumor-Enhancement Visualization and Morphometric Assessment: A Comparison of MPRAGE, SPACE, and VIBE MRI Techniques
}

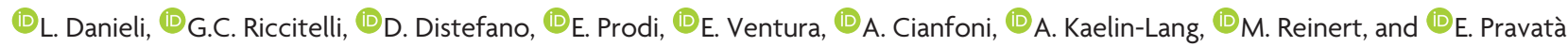
$x \equiv$

\begin{abstract}
BACKGROUND AND PURPOSE: Postgadolinium MR imaging is crucial for brain tumor diagnosis and morphometric assessment. We compared brain tumor enhancement visualization and the "target" object morphometry obtained with the most commonly used 3D MR imaging technique, MPRAGE, with 2 other routinely available techniques: sampling perfection with application-optimized contrasts by using different flip angle evolutions (SPACE) and volumetric interpolated brain examination (VIBE).
\end{abstract}

MATERIALS AND METHODS: Fifty-four contrast-enhancing tumors (38 gliomas and 16 metastases) were assessed using MPRAGE, VIBE, and SPACE techniques randomly acquired after gadolinium-based contrast agent administration on a 3T scanner. Enhancement conspicuity was assessed quantitatively by calculating the contrast rate and contrast-to-noise ratio, and qualitatively, by consensus visual comparative ratings. The total enhancing tumor volume and between-sequence discrepancy in the margin delineation were assessed on the corresponding 3D target objects contoured with a computer-assisted software for neuronavigation. The Wilcoxon signed rank and Pearson $\chi^{2}$ nonparametric tests were used to investigate between-sequence discrepancies in the contrast rate, contrast-to-noise ratio, visual conspicuity ratings, tumor volume, and margin delineation estimates. Differences were also tested for ID (Response Evaluation Criteria in Solid Tumors) and 2D (Response Assessment in Neuro-Oncology) measurements.

RESULTS: Compared with MPRAGE, both SPACE and VIBE obtained higher contrast rate, contrast-to-noise ratio, and visual conspicuity ratings in both gliomas and metastases ( $P$ range, $<.001-.001)$. The between-sequence 3D target object margin discrepancy ranged between $3 \%$ and $19.9 \%$ of lesion tumor volume. Larger tumor volumes, 1D and 2D measurements were obtained with SPACE (P range, <.01-.007).

CONCLUSIONS: Superior conspicuity for brain tumor enhancement can be achieved using SPACE and VIBE techniques, compared with MPRAGE. Discrepancies were also detected when assessing target object size and morphology, with SPACE providing more accurate estimates.

ABBREVIATIONS: CEL = contrast-enhancing lesion; GBCA = gadolinium-based contrast agent; RANO = Response Assessment in Neuro-Oncology; RECIST = Response Evaluation Criteria in Solid Tumors; SPACE = sampling perfection with application-optimized contrasts using different flip angle evolution; TV = tumor volume; $\mathrm{VIBE}=$ volumetric interpolated brain examination

$\mathrm{P}$ ostcontrast MR imaging plays a fundamental role in brain tumor diagnosis, anatomic delineation, and treatment re-

Received December 18, 2018; accepted after revision May 8, 2019.

From the Departments of Neuroradiology (L.D., D.D., E.P., E.V., A.C., E.P.), Neurology (G.C.R., A.K.-L.), and Neurosurgery (M.R.), Neurocenter of Southern Switzerland, Lugano, Switzerland; Neuroimaging Research Unit (G.C.R.), Institute of Experimental Neurology, Division of Neuroscience, San Raffaele Scientific Institute, Vita-Salute San Raffaele University, Milan, Italy; Departments of Neuroradiology (A.C.) and Neurology (A.K.-L.), Inselspital, Bern University Hospital, University of Bern, Bern, Switzerland; and Faculty of Biomedical Sciences (A.K.-L., M.R.), Università della Svizzera Italiana, Lugano, Switzerland.

Lucia Danieli received a grant from the Scientific Research Advisory Board of the Ente Ospedaliero Cantonale (grant number 107.99501) for conducting this study.

Paper previously presented as an oral communication at: European Congress of Radiology, February 28 to March 2, 2018, Vienna, Austria; and Annual Meeting of the American Society of Neuroradiology and the Foundation of the ASNR Symposium, Vancouver, British Columbia, Canada, June 2-7, 2018.

sponse. 3D sequences obtained after gadolinium-based contrast agent (GBCA) injection can illustrate BBB disruption in brain tumors. ${ }^{1-3}$ They can also provide $3 \mathrm{D}$ spatial representations of the enhancing part of the tumor for guiding treatment, biopsy planning, and/or allowing a precise dose delivery during stereotactic radiosurgery. ${ }^{4-7}$ However, various MR imaging techniques can be chosen, each with inherently different characteristics. The most used post-GBCA sequence is MPRAGE, an inversion recovery fast gradient recalled-echo sequence. ${ }^{8,9}$ This technique emphasizes the anatomic brain tissue contrast between gray and white matter

Please address correspondence to Emanuele Pravatà, MD, Via Tesserete 46, 6900, Lugano, Switzerland; e-mail: emanuele.pravata@eoc.ch

$\equiv$ Indicates article with supplemental on-line tables.

Indicates article with supplemental on-line photos.

http://dx.doi.org/10.3174/ajnr.A6096 
due to the inversion recovery preparation pulse. It was recommended for use in brain tumor clinical trials, in both the "Consensus Recommendations for a Standardized Brain Tumor Imaging Protocol in Clinical Trials" ${ }^{10}$ and in the modified Response Assessment in Neuro-Oncology (RANO) criteria. ${ }^{9}$ However, MPRAGE images exhibit suboptimal postgadolinium enhancement visualization, particularly in lesions with low gadolinium concentration. ${ }^{8,11,12}$ On the other hand, in previous investigations, 2 alternative techniques available for clinical use, the sampling perfection with application-optimized contrasts using different flip-angle evolution (SPACE sequence; Siemens, Erlangen, Germany) and the volumetric interpolated brain examination (VIBE) demonstrated a higher sensitivity to enhancing brain lesions. ${ }^{13-17}$ SPACE is a $3 \mathrm{D}-\mathrm{TSE}$ technique ${ }^{18}$ that intrinsically provides higher sensitivity to low gadolinium concentrations and is more resilient to the effects of tumoral hemosiderin and/or calcium deposits, which may reduce enhancement visualization. ${ }^{5,13,18,19}$ VIBE is a fast gradient recalledecho T1-weighted sequence without an inversion recovery preparation pulse, originally used in brain imaging for contrast-enhanced MR imaging venography studies, which use a partial $k$-space acquisition with a "centric-ordering" filling, to obtain both time-efficient and contrast-enhancement sensitive images. ${ }^{16}$

Awareness about MR imaging sequence-related differences in the sensitivity to GBCA enhancement may be particularly useful to decide the most appropriate sequence to use in cases in which a GBCA dose reduction is considered, such as in pregnant women and/or other vulnerable patient populations. ${ }^{20-24}$ In this study, we hypothesized the following: 1) Compared with MPRAGE, SPACE and VIBE may provide superior tumor-enhancement visualization, and 2) morphometric discrepancies may occur among these sequences when contouring the corresponding contrast-enhancing lesions (CELs). We evaluated 54 tumors, comprising both gliomas and metastases amenable to surgical interventions and/or stereotactic radiosurgery, comparing MPRAGE with SPACE and VIBE images with respect to the following: 1) tumor-enhancement contrast rate and contrast-to-noise ratio and visual conspicuity, and 2) tumor volume (TV) and margin delineation of the 3D "target" objects created for treatment planning. As an additional aim, we tested potential differences when using 1D (Response Evaluation Criteria in Solid Tumors [RECIST] ${ }^{25}$ ) and 2D $\left(\mathrm{RANO}^{9,26}\right)$ standard measurements for treatment-response assessment. Finally, the number of metastases not visualized on MPRAGE images was recorded.

\section{MATERIALS AND METHODS}

This study was approved by the local ethics committee of Canton Ticino after obtaining written informed consent from all patients.

Between April 2015 and May 2017, one hundred ninety-six consecutive adult patients undergoing brain MR imaging for suspected or known tumor or tumor relapse following an operation were prospectively enrolled and examined on a 3T scanner (Magnetom Skyra; Siemens) with a 20-channel head coil. Inclusion criteria were the following: enhancing intra-axial tumors that were candidates for neuronavigation-assisted biopsy, surgical removal, and/or stereotactic radiosurgery treatment based on the literature recommendations ${ }^{27-29}$ and multidisciplinary tumor board consensus. Forty-one patients with intra-axial tumors were reviewed; of these, 3 were excluded for movement artifacts, and 1 for incomplete protocol acquisition. In patients with multiple discrete foci of enhancement separated by nonenhancing parenchyma, these were considered individual lesions. Three additional metastases, which were not detected on MPRAGE (see also "Visual Conspicuity" below), were not included in the subsequent analyses. Fifty-four CELs (38 gliomas in 29 patients, and 16 metastases in 8 patients) were finally included. In 38/38 gliomas and $10 / 16$ metastases, the final diagnosis was based on pathology. The diagnoses for the remaining lesions were based collectively on imaging characteristics, clinical history, evidence of remote (nonbrain) malignancy, and MR imaging follow-up. In patients with tumor relapse (16 gliomas), differentiating tumor from treatment-related enhancement was based on perfusion and diffusion imaging, morphology (nodular/expansile versus linear/nonspecific), and sequential MR imaging follow-up.

\section{MR Imaging Sequences}

For MPRAGE and SPACE, we adapted the sequence default parameters recommended by the vendor, including a sagittally oriented slab with a symmetric $k$-space acquisition in all encoding directions, resulting in $1-\mathrm{mm}^{3}$ isotropic voxels. For VIBE, which uses an asymmetric $k$-space acquisition, ${ }^{16}$ we obtained sagittally oriented slabs with a $1 \times 1 \times 1.27 \mathrm{~mm}$ actual voxel size, resulting into a $1-\mathrm{mm}$ isotropic voxel after zerofilling interpolation. To minimize the potential bias related to the time gap between the first and last acquired images after injection, we reduced the scan duration using parallel imaging. ${ }^{30}$ As previously proposed, ${ }^{17,31}$ a relatively higher acceleration factor was used for SPACE $(\times 4)$, to account for the longer acquisition time. Finally, because patients had to be investigated for metastases potentially occurring in nonbrain tissues, fat suppression was also applied to improve contrast enhancement at this level. Scan durations were 1 minute 57 seconds for VIBE, 3 minutes 10 seconds for SPACE, and 3 minutes 46 seconds for MPRAGE. A summary of the main parameters of the sequences is reported in On-line Table 1.

All patients underwent our standard brain tumor MR imaging protocol, including FLAIR, DWI, T2 ${ }^{*}$, T2 TSE, and PWI. For the precontrast T1-weighted images, 1 sequence was randomly chosen among MPRAGE, SPACE, and VIBE. A constant dose of 0.1 $\mathrm{mL} / \mathrm{Kg}$ of gadobutrol, followed by a saline flush, was administered IV in all examinations. Finally, MPRAGE, SPACE, and VIBE sequences were consecutively performed in a randomized order, starting 5 minutes after contrast injection.

\section{Image Analysis}

Quantitative Conspicuity. Two measures, the contrast rate and contrast-to-noise ratio, were calculated with previously used formulas:

$$
\begin{aligned}
& \text { Contrast Rate }=\left[\left(\mathrm{SI}_{\text {lesion }}-\mathrm{SI}_{\text {parenchyma }}\right) / \mathrm{SI}_{\text {parenchyma }}\right] \times 100,{ }^{13,32} \\
& \text { Contrast-to-Noise Ratio }=\left(\mathrm{SI}_{\text {lesion }}-\mathrm{SI}_{\text {parenchyma }}\right) / \mathrm{SD}_{\text {parenchyma }}{ }^{17,31}
\end{aligned}
$$

Where $\mathrm{SI}_{\text {lesion }}$ and $\mathrm{SI}_{\text {parenchyma, }}$, respectively, represent the CEL and the adjacent parenchyma average signal intensity, and $\mathrm{SD}_{\text {parenchyma }}$ represents the standard deviation in $\mathrm{SI}_{\text {parenchyma. }}$ As AJNR Am J Neuroradiol 40:1140-48 Jul 2019 www.ajnr.org 1141 
previously suggested, ${ }^{17,31}$ because parallel imaging was used, SI and SD for noise calculation were taken in the adjacent parenchyma, instead of background air. By means of iPlan, Version 3.0 (Brainlab, Munich, Germany), ROIs were drawn by an expert neuroradiologist (L.D., with 7 years of experience in neuroimaging). For $\mathrm{SI}_{\text {lesion, }}$ ROIs were drawn distant from lesion edges to avoid partial volume effects (average ROI size $=11.88$ $\mathrm{mm}^{2}$ ). For $\mathrm{SI}_{\text {parenchyma }}$, a relatively larger ROI (average size $=$ $74.71 \mathrm{~mm}^{2}$ ) was placed in the adjacent parenchyma, including the gray and white matter to account for signal intensity differences among the tissues, ${ }^{13}$ and avoiding CSF and vessels.

Visual Conspicuity. As previously noted, ${ }^{13,17}$ it is not possible to perform a visual evaluation blinded to the sequence type because expert readers would easily recognize the distinguishing features of the various sequences. Here, 1 neuroradiologist (E.P.) and 1 neurosurgeon (M.R.) with 10 and 15 years of experience, respectively, unaware of the clinical data and quantitative results, assessed all 3 sequence types in each patient. The 3 sequences were presented sequentially for each patient, in a random order, using the Brainlab DICOM viewer. They provided a consensus visual score, aimed at comparing the quality of CEL conspicuity among sequences in a competitive manner and ranking ("best," "intermediate," and "worst"), taking into account the following: 1) enhancement conspicuity, 2) clarity of CEL separation from adjacent vessels, and 3) impact of artifacts from paramagnetic deposits from hemorrhage/calcifications. When the overall visual conspicuity of a given lesion was assessed as similar between 2 or among all 3 sequences, the same ranking was assigned to each. Finally, during a separate session, the number of small metastases visible on SPACE and/or VIBE, but not on MPRAGE images, was retrospectively recorded.

Volume and Margin Delineation. Analyses were performed using a semiautomated tool based on a region-growing algorithm (SmartBrush 2.5; Brainlab). This is previously validated software that demonstrated excellent reliability for brain tumor segmentation. ${ }^{33,34}$ All segmentations were performed by a single neuroradiologist (L.D.) to avoid interrater bias. ${ }^{34}$ After image coregistration among all sequences, they were presented independently to the reader. Region-growing 2D segmentations of the CELs were drawn on perpendicular slices and automatically $3 \mathrm{D}$-interpolated by the software. Then, margin refinements were manually performed with the help of the region-growing algorithm. ${ }^{33}$

Within-sequence reproducibility was performed by repeating segmentations in a randomly chosen subset of 27 (50\%) CELs during a subsequent separate session and by calculating the corresponding intraclass correlation coefficients with a 2-way mixed consistency, average-measured approach. ${ }^{33,35}$ Consistent with previous validation studies, ${ }^{33}$ intraclass correlation coefficient values were excellent: 0.998 for MPRAGE, 95\% CI, 0.995-0.999; 0.997 for VIBE, 95\% CI, 0.993-0.999; and 0.999, 95\% CI, 0.9970.999 for SPACE.

The potential discrepancy occurring between the objects contoured using the different techniques was further assessed concerning lesion margin spatial mismatch. Using the Brainlab object manipulation tool, objects were reciprocally subtracted: 1)
$\left.\left.\left|\mathrm{CEL}_{\text {MPRAGE }}\right|-\left|\mathrm{CEL}_{\mathrm{SPACE}}\right| ; 2\right)\left|\mathrm{CEL}_{\mathrm{SPACE}}\right|-\left|\mathrm{CEL}_{\mathrm{MPRAGE}}\right| ; 3\right)$ $\left|\mathrm{CEL}_{\text {MPRAGE }}\right|-\left|\mathrm{CEL}_{\text {VIBE }}\right|$; and 4) $\left|\mathrm{CEL}_{\mathrm{VIBE}}\right|-\left|\mathrm{CEL}_{\text {MPRAGE }}\right|$. We defined the resulting differences as margin extent discrepancy. This quantified the mismatch volume of the regions where a given sequence exceeded another and vice versa (see the explanatory diagram in Fig 1). The amount of mismatch with respect to the entire CEL was assessed by calculating the ratio (expressed in percentages) between the margin extent discrepancy and the median MPRAGE TV as a reference.

ID and 2D Measurements. In a subsequent session, 3 expert neuroradiologists (L.D., D.D., E.P.), with 7, 8, and 10 years of experience in brain tumor imaging, respectively, each independently measured the longest CEL diameter (1D RECIST ${ }^{25}$ ) and the product of the 2 longest perpendicular diameters (2D RANO ${ }^{9}$ ), excluding cystic cavities and lesions with poorly defined margins. Only lesions of diameter $\geq 10 \mathrm{~mm}$ were considered measurable. Twenty-nine CELs (22 gliomas, 7 metastases) were evaluated and randomly presented to the readers, while varying sequences and patients. Finally, for each lesion, the 3 readers' measurements were averaged to perform sequence comparisons.

\section{Statistical Analysis}

All computations were performed using the SPSS software package, Version 22.0 (IBM, Armonk, New York). Normal distribution assumptions were checked by Kolmogorov-Smirnov and Shapiro-Wilk tests. Descriptive analyses for quantitative variables used median and interquartile range, while frequencies were used for the qualitative measures. In all CELs, as well as gliomas and metastases separately, differences between pairs of sequences in the contrast rate, contrast-to-noise ratio, and TV were investigated using the nonparametric Wilcoxon test. The visually estimated conspicuity ranking scores among sequences were compared using the $\chi^{2}$ test. Bland-Altman diagrams were performed to represent the agreement of TV measures between sequences, and the corresponding repeatability coefficients were calculated. ${ }^{36,37}$ As for $1 \mathrm{D}$ and $2 \mathrm{D}$ measurements, given the limited sample size, analyses were performed in all CELs only. Interreader reproducibility of $1 \mathrm{D}$ and $2 \mathrm{D}$ measurements was estimated using the intraclass correlation coefficient.

The FSL General Linear Model (http://fsl.fmrib.ox.ac.uk/fsl/ fslwiki/GLM) for repeated measures was used to adjust for the potential confound effect of the acquisition order between compared sequences, with respect to the contrast rate, contrast-tonoise ratio, TV, and 1D and 2D differences on Poisson distribution. The General Linear Model was also applied to test the potential confound of postsurgical and posttreatment tissue changes, which can influence the enhancement intensity at different CEL sites. ${ }^{38}$ This potential confounding factor was addressed through testing the interaction effect of recurrence (as a binomial variable) on TV differences with the General Linear Model. The level of significance was set at $P<.05$.

\section{RESULTS}

Of 57 CELs detected by SPACE, 3 small ( $\leq 2$-mm diameter) metastases (5.3\% of all lesions, $15.8 \%$ of all metastases) were missed 


\section{CEL contouring}

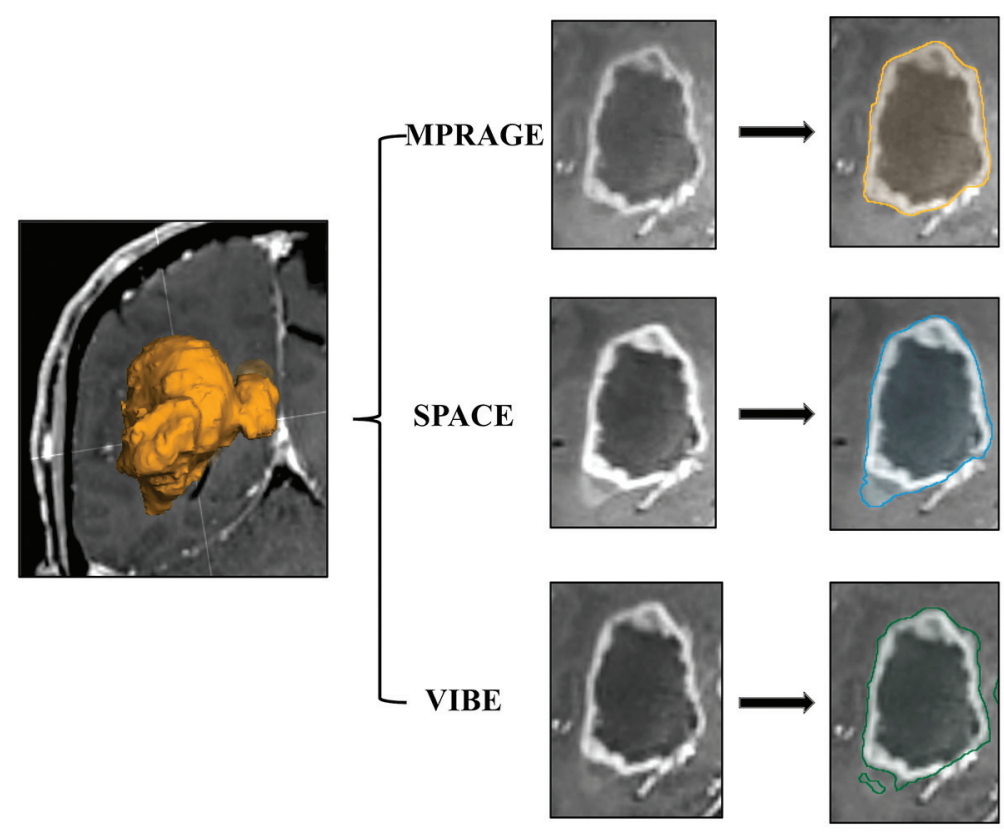

A
MED Calculation
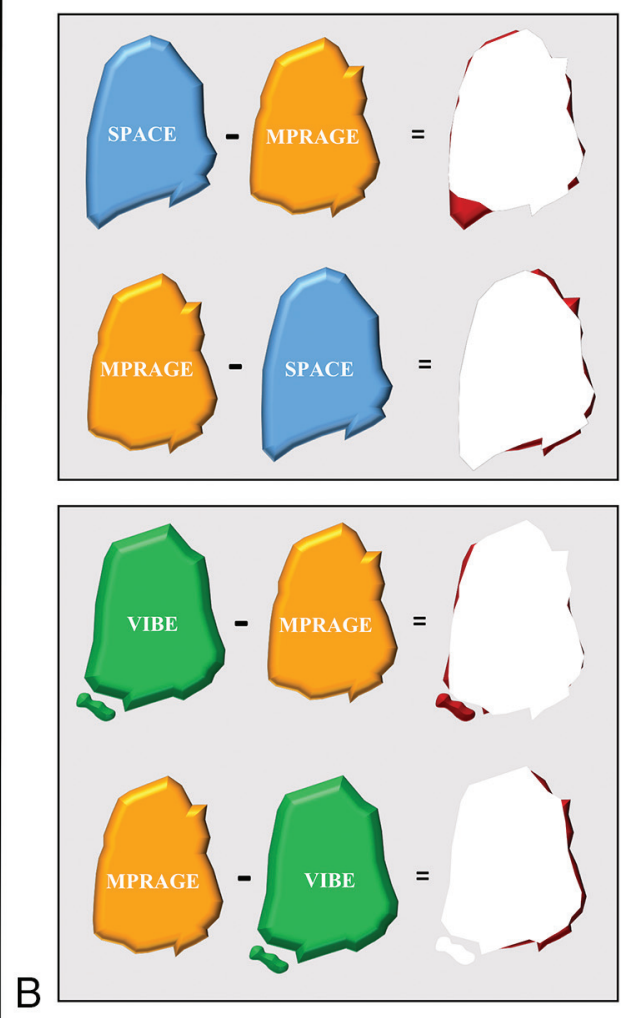

FIG 1. Diagram showing the contrast-enhancing lesion margin extent discrepancy (MED) estimation procedure. This approach is aimed at highlighting the spatial mismatch of the tumor border segmentation obtained from MPRAGE, with respect to SPACE and VIBE, and vice versa. $A$, First, for each CEL and sequence type, volume segmentation is performed using a validated computer-assisted tool dedicated to pretreatment planning and neuronavigation (SmartBrush 2.5; Brainlab). Segmentations obtained on MPRAGE, SPACE, and VIBE images are, respectively, represented in orange, blue, and green. $B$, The segmented volumes are reciprocally subtracted, generating maps of the areas where SPACE and VIBE volumes, respectively, exceed MPRAGE, and vice versa. Finally, the resulting MED areas are represented in red.

Table 1: Quantitative and qualitative conspicuity assessment

\begin{tabular}{|c|c|c|c|c|c|}
\hline & \multirow[b]{2}{*}{ CR Median (IQR) } & \multirow[b]{2}{*}{ CNR Median (IQR) } & \multicolumn{3}{|c|}{ Visual Score } \\
\hline & & & Best $^{a}$ & Intermediate & Worst \\
\hline \multicolumn{6}{|l|}{ All CELs $(n=54)$} \\
\hline 3D-IR GRE MPRAGE & $80.88(43.71-125.65)$ & 10.02 (5.71-16.06) & $15(27.8 \%)$ & $26(48.1 \%)$ & $13(24.1 \%)$ \\
\hline 3D-TSE SPACE & $100.92^{\mathrm{b}, \mathrm{c}}(73-191.59)$ & $19.17^{\mathrm{b}, \mathrm{c}}(13.21-36.01)$ & $54(100 \%)$ & 0 & 0 \\
\hline 3D-GRE VIBE & $85.86^{\mathrm{b}, \mathrm{c}}(43.71-135.22)$ & $16.76^{\mathrm{b}, \mathrm{c}}(11-37.22)$ & $24(44.4 \%)^{\mathrm{b}}$ & 25 (46.3\%) & $5(9.3 \%)^{b}$ \\
\hline \multicolumn{6}{|l|}{ Gliomas $(n=38)$} \\
\hline 3D-IR GRE MPRAGE & 90.01 (48.29-125.21) & $10.51(5.68-17.33)$ & $9(23.7 \%)$ & $19(50 \%)$ & $10(26.3 \%)$ \\
\hline 3D-TSE SPACE & $125.64^{\mathrm{b}, \mathrm{c}}(88.41-187.45)$ & $24.46^{\mathrm{b}, \mathrm{c}}(12.55-41.23)$ & 38 (100\%) & 0 & 0 \\
\hline 3D-GRE VIBE & $94.19^{\mathrm{b}, \mathrm{c}}(58.34-88.95)$ & $16.22^{d, c}(9.89-27.25)$ & $16(42.1 \%)^{d}$ & $18(47.4 \%)$ & $4(10.5 \%)^{d}$ \\
\hline \multicolumn{6}{|l|}{ Metastases $(n=16)$} \\
\hline 3D-IR GRE MPRAGE & 54.71 (35.24-130.01) & 9.30 (6.77-12.29) & $6(37.5 \%)$ & $7(43.8 \%)$ & $3(18.8 \%)$ \\
\hline 3D-TSE SPACE & $80.04^{\mathrm{b}, c}(63.13-200)$ & $17.15^{\mathrm{b}, \mathrm{c}}(13.63-21.26)$ & $16(100 \%)$ & 0 & 0 \\
\hline 3D-GRE VIBE & $72.95^{\mathrm{b}, \mathrm{c}}(54.19-154.43)$ & $17.26^{\mathrm{b}, \mathrm{c}}(12.14-31.18)$ & $8(50 \%)$ & $7(43.8 \%)$ & $1(6.3 \%)$ \\
\hline
\end{tabular}

Note:-IR indicates inversion recovery; GRE, gradient recalled-echo; CR, contrast rate; CNR, contrast-to-noise ratio; IQR, interquartile range.

a Because SPACE in the visual assessment was constantly rated as best in all cases, no statistical comparison tests were performed in this analysis.

b $P<.001$.

${ }^{c} P \leq .01$ (adjusted for the order of sequence acquisition; FSL General Linear Model).

${ }^{\mathrm{d}} P=.001$ (compared with MPRAGE, Wilcoxon test).

on MPRAGE. The remaining 54 CELs were included in the subsequent analyses. Patients' main demographics and CEL characteristics are presented on On-line Table 2.

\section{Conspicuity Assessment}

Both the contrast rate and contrast-to-noise ratio were significantly higher on either SPACE and VIBE images compared with MPRAGE, in all groups. Differences remained significant after adjusting for the acquisition order of the compared sequences (Table 1). For visual assessment, in all CELs, MPRAGE obtained the best ranking in $15 / 54(27.8 \%)$, intermediate in $26 / 54$ (48.1\%), and worst in $13 / 54(24.1 \%)$. SPACE provided the best CEL visualization in all cases $(54 / 54,100 \%)$; therefore, no statistical comparison was performed with MPRAGE. VIBE obtained a higher frequency of best $(24 / 54,44.4 \%)$ and a lower frequency of worst 
ratings $(5 / 54,9.3 \%)$ compared with MPRAGE $(P<.001)$, whereas the frequency of intermediate ratings was not significantly different $(25 / 54,46.3 \%)$. Similar results were obtained by VIBE in the glioma subgroup $(P=.001)$, while no significant differences were found in metastases subgroup. Two illustrative cases, with an example of a tiny metastasis not visible on MPRAGE images, are presented in Fig 2.
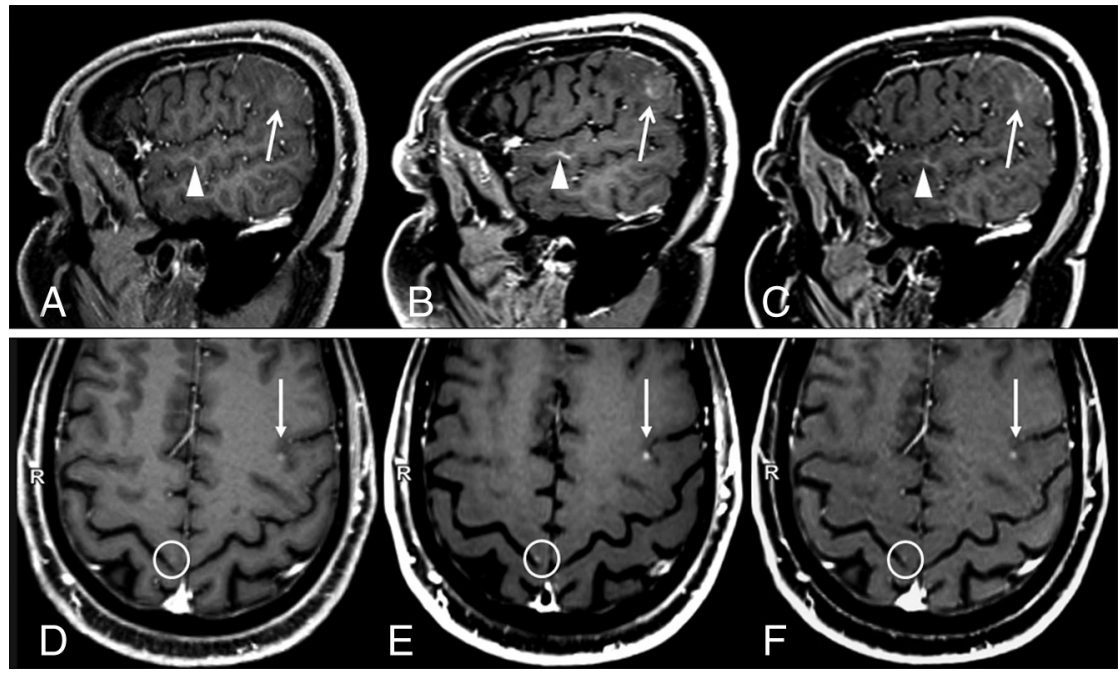

FIG 2. Two sample cases showing differences in contrast-enhancing lesion conspicuity between sequences. $A$ and $D$, MPRAGE. $B$ and $E$, SPACE. $C$ and $F$, VIBE. $A-C$, Case 1 : a patient with a faintly enhancing glioblastoma. Compared with MPRAGE ( $A$ ), the lesion enhancement (arrows) and its boundary demarcations are much better appreciated on SPACE and VIBE images. The corresponding contrast rate/contrast-to-noise ratio values are 24.75/2.45, 51.32/8.96, and 41.23/6.25, and the rankings are worst, best, and intermediate, respectively, for MPRAGE, SPACE, and VIBE. Also incidentally noted is a developmental venous anomaly (arrowheads), which shows a strong contrast enhancement on black-blood SPACE images. This is probably related to the extremely slow flow seen in such small venous malformations. Images were acquired at 5 minutes after contrast injection in the following order: VIBE, SPACE, MPRAGE. $D-F$, Case 2: a patient with metastases from renal carcinoma ( $D$, MPRAGE. E, SPACE. F, VIBE). A small CEL is seen in the left frontal lobe (arrows) whose conspicuity with respect to the background parenchyma was ranked worst on MPRAGE, intermediate on VIBE, and best on SPACE images. The corresponding contrast rate/contrast-to-noise ratio values are 8.85/3.08, 18.96/9.15, and 16.63/6.89, respectively. An example of a very tiny metastasis in the right precentral gyrus cortex, which was missed when inspecting MPRAGE images alone but was visible on SPACE and VIBE, is highlighted by circles. This lesion was not included in the analyses. Images were acquired after 5 minutes from contrast injection in the following order: SPACE, VIBE, and MPRAGE.

\section{Volume and Margin Delineation Assessment}

ween-sequence TV and margin extent discrepancy estimation volumes. The corresponding measure agreement distribution is illustrated by the Bland-Altmann plots in On-line Fig 1 . The repeatability coefficient was $2.03 \mathrm{~cm}^{3}$. After adjusted for the compared sequenceacquisition order, differences remained significant in all CELs and gliomas and approached significance for metastases $(P=.075)$ (Table 2). No significant interaction effect of recurrence (previously treated lesions) was detected $(F=$ 0.958, $P=.332$ ). No discrepancy was found between MPRAGE and VIBE in all groups ( $P$ range, .201-.343).

The margin extent discrepancy analysis results are reported in Table 2. As for the $\left|\mathrm{CEL}_{\text {MPRAGE }}\right|-\left|\mathrm{CEL}_{\text {SPACE }}\right|$ comparison (quantifying the areas where MPRAGE exceeded SPACE margins), the margin extent discrepancy represented 7.4\% (all lesions), 6\% (gliomas), and $6.1 \%$ (metastases) of the TV, whereas for the reciprocal $\left|\mathrm{CEL}_{\mathrm{SPACE}}\right|-$ $\mid$ CEL $_{\text {MPRAGE }}$ subtraction (quantifying the areas where SPACE exceeded MPRAGE margins), the margin extent discrepancy was 19.9\% (all lesions), $14.2 \%$ (gliomas), and $15.2 \%$ (metastases). Regarding the $\left|\mathrm{CEL}_{\text {MPRAGE }}\right|-$ $\left|\mathrm{CEL}_{\mathrm{VIBE}}\right|$ mismatch, the margin extent discrepancy was $11 \%$ (all lesions), 7\% (gliomas), and 3\% (metastases) of the $\mathrm{TV}$, whereas for the $\left|\mathrm{CEL}_{\mathrm{VIBE}}\right|-$ $\mid$ CEL $_{\text {MPRAGE }} \mid$ mismatch, it was $11 \%$ (all lesions), $7.6 \%$ (gliomas), and

Table 2: Morphometric assessment

\begin{tabular}{|c|c|c|c|}
\hline & All CELs $(n=54)$ & Gliomas $(n=38)$ & Metastases $(n=16)$ \\
\hline \multicolumn{4}{|l|}{ TV (median/IQR) (mL) } \\
\hline MPRAGE & $1.36 / 0.18-10.93$ & $3.02 / 0.44-28.93$ & $0.33 / 0.006-2.81$ \\
\hline SPACE & $1.78 / 0.20-11.00$ & $3.5 / 0.49-29.73$ & $0.39 / 0.006-3.2$ \\
\hline VIBE & $1.62 / 0.16-10.35$ & $3.39 / 0.43-27.48$ & $0.40 / 0.007-2.84$ \\
\hline \multicolumn{4}{|l|}{ TV, SPACE vs MPRAGE } \\
\hline$P^{a}$ & $.001^{\mathrm{b}}$ & $.007^{\mathrm{b}}$ & $.003^{b}$ \\
\hline$P$ (adjusted for sequence acquisition order) ${ }^{c}$ & $.034^{\mathrm{b}}$ & $.033^{\mathrm{b}}$ & .075 \\
\hline \multicolumn{4}{|l|}{ TV, VIBE vs MPRAGE } \\
\hline$P^{a}$ & .259 & .201 & .343 \\
\hline$P$ (adjusted for sequence acquisition order) $^{c}$ & .521 & .538 & .706 \\
\hline \multicolumn{4}{|l|}{ MED (median/\% of TV) } \\
\hline $\mid$ CEL $_{\text {MPRAGE }}|-|$ CEL $_{\text {SPACE }} \mid$ & $0.10 \mathrm{~mL} / 7.4 \%$ & $0.18 \mathrm{~mL} / 6 \%$ & $0.02 \mathrm{~mL} / 6.1 \%$ \\
\hline$\left|\mathrm{CEL}_{\mathrm{SPACE}}\right|-\left|\mathrm{CEL}_{\mathrm{MPRAGE}}\right|$ & $0.27 \mathrm{~mL} / 19.9 \%$ & $0.43 \mathrm{~mL} / 14.2 \%$ & $0.05 \mathrm{~mL} / 15.2 \%$ \\
\hline$\left|\mathrm{CEL}_{\text {MPRAGE }}\right|-\left|\mathrm{CEL}_{\mathrm{VIBE}}\right|$ & $0.15 \mathrm{~mL} / 11 \%$ & $0.21 \mathrm{~mL} / 7 \%$ & $0.01 \mathrm{~mL} / 3 \%$ \\
\hline$\left|C_{\text {VIBE }}\right|-\left|C_{\text {MPRAGE }}\right|$ & $0.15 \mathrm{~mL} / 11 \%$ & $0.23 \mathrm{~mL} / 7.6 \%$ & $0.05 \mathrm{~mL} / 15.2 \%$ \\
\hline
\end{tabular}

Note:-MED indicates margin extent discrepancy; IQR, interquartile range.

a Wilcoxon signed rank test.

b Statistically significant differences.

c General Linear Model. 


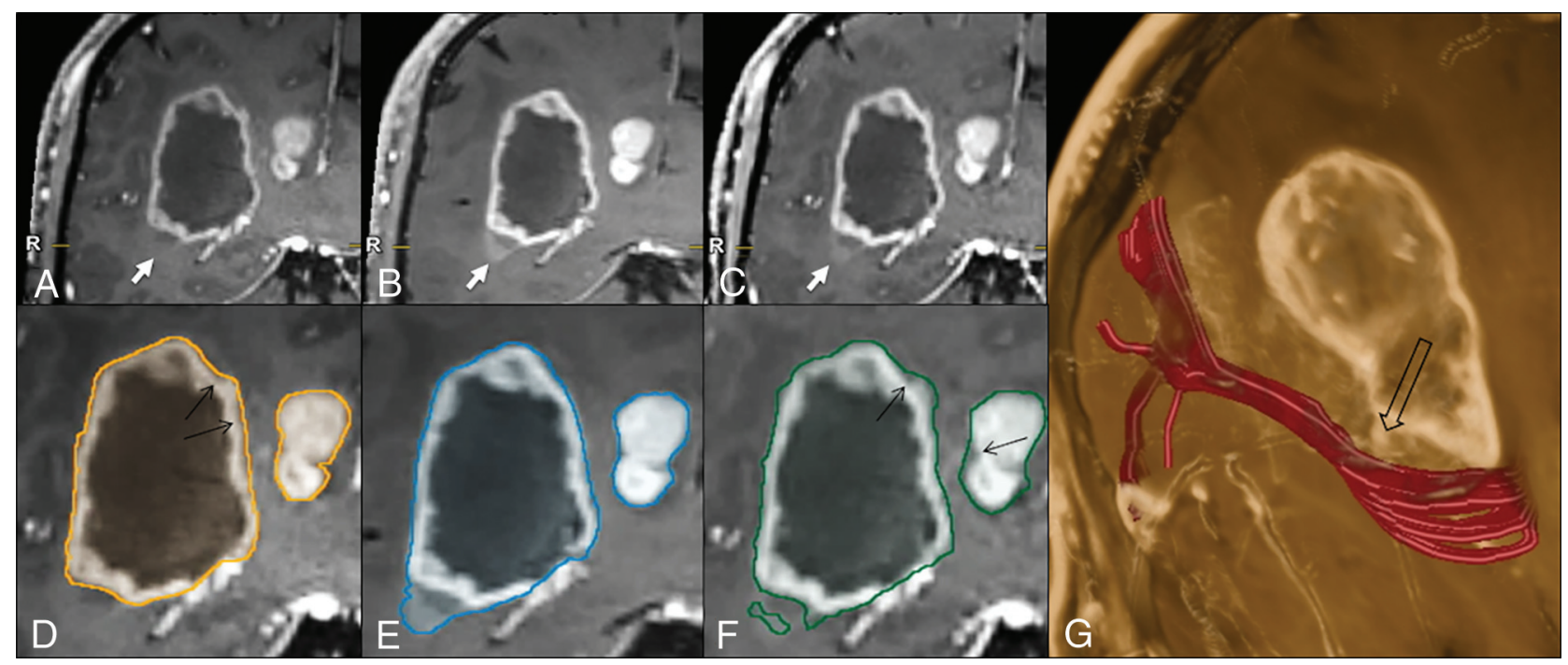

FIG 3. Illustrative case comparing the 3D target-object-creation results in a glioblastoma, obtained on MPRAGE, SPACE, or VIBE images (see the text and Fig 1 for method explanation). There is a clear difference among the MPRAGE (A), SPACE (B), and VIBE (C) conspicuities at the level of the faintly enhancing inferolateral border of the lesion (arrows), which is better represented on the SPACE and VIBE images, compared with MPRAGE. This part of the tumor is not included in the MPRAGE lesion segmentation $(D)$; however, it is captured completely on SPACE (E) and partially on VIBE $(F)$ images. The tractographic reconstruction of the optic radiation trajectory $(G$, in red) demonstrates the close proximity of the tumor to this tract (empty arrow). The black arrows in $D$ and $F$ indicate some thin areas of tumor margin overestimation on MPRAGE and VIBE, respectively, which are not seen on SPACE images. Images were acquired after 5 minutes from contrast injection in the following order: MPRAGE, VIBE, and SPACE.

$15.2 \%$ (metastases). Segmentation examples are provided in Fig 3 and in On-line Fig 2, highlighting sources of mismatch related to differences in the enhancement sensitivity, erroneous margin estimation, and vessel segmentation, as well as susceptibility artifacts.

\section{ID and 2D Assessment}

The interobserver reproducibility, investigated with intraclass correlation coefficient single measurements, showed good agreement among the 3 readers for both 1D (MPRAGE: intraclass correlation coefficient $=0.69,95 \% \mathrm{CI}, 0.56-0.79$; VIBE: intraclass correlation coefficient $=0.73,95 \% \mathrm{CI}, 0.61-0.82$; and SPACE: intraclass correlation coefficient $=0.74,95 \% \mathrm{CI}, 0.63-0.83$ ), and 2D (MPRAGE: intraclass correlation coefficient $=0.74,95 \% \mathrm{CI}$, 0.63-0.83; VIBE: intraclass correlation coefficient $=0.81,95 \%$ CI, 0.72-0.88; and SPACE: intraclass correlation coefficient $=$ $0.81,95 \%$ CI, 0.72-0.88). At the between-sequence comparison (On-line Table 3), both measurements were significantly discrepant between MPRAGE and SPACE $(P<.001$ and .001 , respectively). Differences were also significant after adjusting for the acquisition order of compared sequences (On-line Table 3). No significant discrepancies with VIBE images were found.

\section{DISCUSSION}

Contrast enhancement refers to hyperintensity on T1-weighted images following GBCA administration, which is the result of extravasation of gadolinium-containing macromolecules through a leaky BBB. GBCA accumulates within tumor interstitium, resulting in longitudinal relaxation time shortening and consequent $\mathrm{T} 1$ signal hyperintensity. ${ }^{1}$ Contrast enhancement helps CEL detection, characterization, and pretreatment anatomic assessment and represents a biomarker of potential high-grade tumor within malignant gliomas. ${ }^{2,3,6,39}$ A number of factors are known to in- fluence the CEL conspicuity with respect to the background parenchyma: the $\mathrm{BBB}$ ultrastructural characteristics, ${ }^{40}$ magnetic field strength, ${ }^{41}$ GBCA concentration, relaxivity properties, time from injection, and MR imaging technique. Here, we quantitatively and qualitatively show that tumor-enhancement visualization with SPACE and VIBE is superior to that in MPRAGE, the currently recommended standard technique for brain tumor clinical trials. ${ }^{9,10}$ In this study, SPACE and VIBE demonstrated significantly superior visual conspicuity ratings across all the CEL assessments, with SPACE having the best enhancement visualization in $100 \%$ of cases and VIBE in $44 \%$, compared with $27.8 \%$ for MPRAGE. Conversely, MPRAGE received the worst ranking among sequences in $24.1 \%$ of all CELs, compared with only $9.3 \%$ for VIBE. On image inspection, we found that subtly enhancing parts of the tumors detected by SPACE and VIBE may be crucially missed by MPRAGE. Accordingly, 3 very small metastases could be detected only by SPACE. These results confirm and extend previous investigations conducted on metastatic lesions. Kato et $\mathrm{al}^{13}$ and Komada et $\mathrm{al}^{42}$ documented higher contrast rate and contrast-to-noise ratio estimated by SPACE. In addition, a retrospective study by Kammer et $\mathrm{al}^{17}$ showed that the use of SPACE allowed increased sensitivity and diagnostic confidence in the detection of small lesions. Previous information about VIBE performance was remarkably scarce: only 1 previous study conducted at $1.5 \mathrm{~T}$ by Wetzel et $\mathrm{al}^{16}$ reported higher contrast-to-noise ratio values and visual conspicuity in a heterogeneous group of brain tumors, compared with MPRAGE.

To the best of our knowledge, this is the first study providing information about morphometric discrepancies occurring with brain tumor segmentation, related to sequence-enhancement conspicuity differences. First, we found that discrepant and significantly larger TV estimates were obtained using SPACE com- 
pared with MPRAGE, both in all lesions and in gliomas and metastases considered separately. However, the use of VIBE images did not lead to significantly different TV estimates with respect to MPRAGE. Second, consistent with the larger TV estimates, SPACE contours exceeded those created with MPRAGE by $12.8 \%$ of the TV in all CELs. Most important, despite providing smaller TV estimates, MPRAGE margins exceeded SPACE margins by $6.2 \%$ of the TV, as well. Factors contributing to such a spatial delineation discrepancy included the different techniques sensitive to subtle enhancement, overestimation of tumor borders, erroneous vessel contouring, and/or interference from hemorrhagic/calcium deposit-related susceptibility artifacts (Fig 3 and Online Figs 2 and 3). Although an in vivo reference standard is not available to establish the CEL TV and morphology, the fact that SPACE images obtained higher contrast rate and contrast-tonoise ratio and visual conspicuity ratings from expert readers ${ }^{43}$ reasonably supports the higher accuracy of this technique compared with MPRAGE for CEL boundary delineation. Furthermore, because high-flow intracranial arteries or venous sinuses appear dark on TSE images due to the flow void effect, they could be more accurately separated from the adjacent CEL than on fast gradient recalled-echo images, which conversely display enhancing vessels due to the flow-related enhancement effect. ${ }^{10,13,17}$

Our findings have several practical implications. First, the use of SPACE and/or VIBE sequences may facilitate the identification of areas with relatively higher grade malignancy within gliomas. This is important for guiding accurate biopsy sampling and consequent correct histologic grading and treatment decision-making. Second, because achieving maximal tumor resection may lead to more favorable patient outcomes and the extent of resection may be increased with image-guided approaches, ${ }^{44}$ optimizing target delineation accuracy facilitates precision surgery in individual patients. In the setting of stereotactic radiosurgery planning, using low-conspicuity images could also hinder targetvolume margin delineation and result in suboptimal dose delivery to all CEL sites and/or in unnecessary dose delivery to surrounding eloquent brain structures. ${ }^{4-7}$ An exemplary case, demonstrating MPRAGE underestimation of tumor margins adjacent to the optic radiation, is presented in Fig 3.

Third, accurate count of brain metastases for radiosurgery planning is important to decide between stereotactic radiosurgery and whole-brain treatment approaches. This importance is underscored by a previous study ${ }^{45}$ that emphasized the use of double-dose GBCA to improve MR imaging lesion-detection sensitivity for treatment planning. In contrast, maximizing image sensitivity to low-GBCA concentrations may play a crucial role whenever dose reduction is deemed appropriate to limit a patient's gadolinium tissue deposition. ${ }^{20-24,41}$ Fourth, the varied postcontrast conspicuity should be taken into account for follow-up examinations when different techniques are used, because this has the potential to confound treatment-response assessment, both in terms of enhancement degree and TV. Finally, our findings lend strength to designing data-acquisition strategies dedicated to MR imaging computer-assisted metastasis detection, ${ }^{46}$ as well as multiparametric diagnostic and prognostic assessment of gliomas, ${ }^{47-50}$ which rely on highly accurate and standardized measures of tumor MR imaging features.
The RECIST ${ }^{25}$ and RANO ${ }^{9,26}$ criteria, which represent standardized methodologies for the assessment of tumor response to treatment, respectively rely on $1 \mathrm{D}$ and 2D CEL measurements. In line with $3 \mathrm{D}$-TV findings, both the $1 \mathrm{D}$ and $2 \mathrm{D}$ estimates performed in all measurable CELs were larger when using SPACE compared with MPRAGE. However, the limited number of measurable lesions available in this study did not allow a specific assessment of glioma and metastasis tumor types. Nevertheless, if confirmed in larger series, the present results might suggest the use of 3D-TSE techniques rather than MPRAGE to improve diagnostic accuracy in the design of clinical trials and in clinical practice.

Because the main goal of the present study was to test whether SPACE and/or VIBE may represent valid alternatives to MPRAGE, a direct comparison between SPACE and VIBE was not performed. However, we found that contrary to SPACE, VIBE did not lead to significant differences of morphometric assessment. We speculate that these differences are due to varied technique characteristics. For example, in SPACE sequences, the magnetization refocusing is obtained by radiofrequency pulses, which increase image resistance to magnetic field inhomogeneity. ${ }^{15}$ Another limitation with VIBE is the use of partial $k$-space acquisition to increase time efficiency ${ }^{16}$ so that the acquired voxel size is larger than the final interpolated size, thus potentially having a blurring effect. In addition, VIBE images show vessel enhancement, which may confound CEL boundary segmentation.

\section{Limitations and Future Perspectives}

While our sample size is relatively small, thorough selection was performed to represent cases potentially encountered in a routine neurosurgical and/or stereotactic radiosurgery treatment setting. The time gap between image acquisition and contrast agent injection represents a potential bias for the comparison of sequences acquired during the same examination because a larger GBCA amount may accumulate in the tumor within this time. However, obtaining data acquisition at exactly the same time for all test sequences would ideally require repeat contrast injections, resulting in an unnecessary dose increase to patients. ${ }^{20,21}$ We limited this bias by the following: 1) randomizing the sequence-acquisition order, 2) starting image acquisition 5 minutes after injection to allow a plateau phase, 3 ) reducing scan duration using parallel imaging, and 4) repeating quantitative analyses after adjusting for the acquisition order of compared sequences. Another potential confounding factor included different characteristics of GBCA uptake within posttreatment change. ${ }^{38}$ In principle, this factor may bias morphometry assessment in previously treated (relapsing) gliomas. However, all these were carefully selected to differentiate tumor from posttreatment-related change, by using perfusion and diffusion imaging, morphology, and sequential MR imaging follow-up. In addition, we detected no significant effect on the between-sequence volume differences. The potential variability across different magnetic field intensities and vendors providing 3D-TSE T1 (eg, CUBE, GE Healthcare, Milwaukee, Wisconsin; VISTA, Philips Healthcare, Best, the Netherlands) and/or 3D-fast gradient recalled echo volume-interpolated T1 (LAVA; General Electric) techniques was not assessed in this study. Finally, we did not focus on reproducibility assessment across dif- 
ferent software tools, or operators performing segmentations. Further investigations are warranted to extrapolate our results to additional treatment centers using different computer-assisted segmentation approaches and varied operator experience levels.

\section{CONCLUSIONS}

SPACE and VIBE postcontrast techniques may provide better visualization of brain tumor enhancement than MPRAGE. Morphometric discrepancies existed among sequences, with SPACE leading to potentially more accurate contouring estimates.

\section{ACKNOWLEDGMENTS}

We would like to thank Dr Rachel Delfanti for help in editing this article. This study received a grant from the Scientific Research Advisory Board of the Ente Ospedaliero Cantonale, Bellinzona, Ticino, Switzerland.

Disclosures: Lucia Danieli—RELATED: Grant: Advisory Board for Research of the Ente Ospedaliero Cantonale, Comments: I received a grant from the Advisory Board for Research of the Hospital Cooperation (Ente Ospedaliero Cantonale) (grant No. 107.99501) for conducting this study. Alain Kaelin-Lang_UNRELATED: Consultancy: Advisory Boards (Merz Pharma, Zambon)*; Employment: Medical and Scientific Director of the Neurocenter of Southern Switzerland; Grants/Grants Pending: Fondazione Svizzera per la Ricerca sulle Malattie Muscolari Switzerland, Swiss Parkinson Biotech; Payment for Lectures including Service on Speakers Bureaus: sponsored lectures (Merz Pharma).* Michael Reinert-UNRELATED: Grants/grants pending: Lega Cancor Ticinese, Gabruele Charitable Foundation, Comments: Neurooncology Research Group. *Money paid to the institution.

\section{REFERENCES}

1. Gadian DG, Payne JA, Bryant DJ, et al. Gadolinium-DTPA as a contrast agent in MR imaging-theoretical projections and practical observations. J Comput Assist Tomogr 1985;9:242-51 CrossRef Medline

2. Healy ME, Hesselink JR, Press GA, et al. Increased detection of intracranial metastases with intravenous Gd-DTPA. Radiology 1987; 165:619-24 CrossRef Medline

3. Sze G, Milano E, Johnson C, et al. Detection of brain metastases: comparison of contrast-enhanced MR with unenhanced MR and enhanced CT. AJNR Am J Neuroradiol 1990;11:785-91 Medline

4. Zhao F, Li M, Kong L, et al. Delineation of radiation therapy target volumes for patients with postoperative glioblastoma: a review. Onco Targets Ther 2016;9:3197-204 CrossRef Medline

5. Cao Y, Tseng CL, Balter JM, et al. MR-guided radiation therapy: transformative technology and its role in the central nervous system. Neuro Oncol 2017;19:ii16-29 CrossRef Medline

6. Wang LL, Leach JL, Breneman JC, et al. Critical role of imaging in the neurosurgical and radiotherapeutic management of brain tumors. Radiographics 2014;34:702-21 CrossRef Medline

7. Farace P, Giri MG, Meliadò G, et al. Clinical target volume delineation in glioblastomas: pre-operative versus post-operative/pre-radiotherapy MRI. Br J Radiol 2011;84:271-78 CrossRef Medline

8. Mugler JP 3rd, Brookeman JR. Theoretical analysis of gadopentetate dimeglumine enhancement in T1-weighted imaging of the brain: comparison of two-dimensional spin-echo and three-dimensional gradient-echo sequences. J Magn Reson Imaging 1993;3:761-69 CrossRef Medline

9. Ellingson BM, Wen PY, Cloughesy TF. Modified criteria for radiographic response assessment in glioblastoma clinical trials. Neurotherapeutics 2017;14:307-20 CrossRef Medline

10. Ellingson BM, Bendszus M, Boxerman J, et al; Jumpstarting Brain Tumor Drug Development Coalition Imaging Standardization Steering Committee. Consensus recommendations for a standardized brain tumor imaging protocol in clinical trials. Neuro Oncol 2015; 17:1188-98 CrossRef Medline

11. Schmilz BL, Aschoff AJ, Hoffmann MH, et al. Advantages and pitfalls in 3T MR brain imaging: a pictorial review. AJNR Am J Neuroradiol 2005;26:2229-37 Medline

12. Blüml S, Schad LR, Scharf J, et al. A comparison of magnetization prepared 3D gradient-echo (MP-RAGE) sequences for imaging of intracranial lesions. Magn Reson Imaging 1996;14:329-35 CrossRef Medline

13. Kato Y, Higano S, Tamura H, et al. Usefulness of contrast-enhanced T1-weighted sampling perfection with application-optimized contrasts by using different flip angle evolutions in detection of small brain metastasis at 3T MR imaging: comparison with magnetization-prepared rapid acquisition of gradient echo imaging.. AJNR Am J Neuroradiol 2009;30:923-29 CrossRef Medline

14. Hodel J, Outteryck O, Ryo E, et al. Accuracy of postcontrast 3D turbo spin-echo MR sequence for the detection of enhanced inflammatory lesions in patients with multiple sclerosis. AJNR Am J Neuroradiol 2014;35:519-23 CrossRef Medline

15. Reichert M, Morelli JN, Runge VM, et al. Contrast-enhanced 3-dimensional SPACE versus MP-RAGE for the detection of brain metastases: considerations with a 32-channel head coil. Invest $R a$ diol 2013;48:55-60 CrossRef Medline

16. Wetzel SG, Johnson G, Tan AGS, et al. Three-dimensional, T1weighted gradient-echo imaging of the brain with a volumetric interpolated examination. AJNR Am J Neuroradiol 2002;23:995-1002 Medline

17. Kammer NN, Coppenrath E, Treitl KM, et al. Comparison of contrast-enhanced modified T1-weighted 3D TSE black-blood and 3D MP-RAGE sequences for the detection of cerebral metastases and brain tumours. Eur Radiol 2016;26:1818-25 CrossRef Medline

18. Mugler JP 3rd, Bao S, Mulkern RV, et al. Optimized single-slab three-dimensional spin-echo MR imaging of the brain. Radiology 2000;216:891-99 CrossRef Medline

19. Chappell PM, Pelc NJ, Foo TK, et al. Comparison of lesion enhancement on spin-echo and gradient-echo images. AJNR Am J Neuroradiol 1994;15:37-44 Medline

20. Ramalho J, Semelka RC, Ramalho M, et al. Gadolinium-based contrast agent accumulation and toxicity: an update. AJNR Am J Neuroradiol 2016;37:1192-98 CrossRef Medline

21. Thomsen HS, Morcos SK, Almen T, et al; ESUR Contrast Medium Safety Committee. Nephrogenic systemic fibrosis and gadoliniumbased contrast media: updated ESUR Contrast Medium Safety Committee guidelines. Eur Radiol 2013;23:307-18 CrossRef Medline

22. Gathings RM, Reddy R, Santa Cruz D, et al. Gadolinium-associated plaques: a new, distinctive clinical entity. JAMA Dermatol 2015;151: 316-19 CrossRef Medline

23. Khawaja AZ, Cassidy DB, Al Shakarchi J, et al. Revisiting the risks of MRI with gadolinium based contrast agents: review of literature and guidelines. Insights Imaging 2015;6:553-58 CrossRef Medline

24. Gong E, Pauly JM, Wintermark M, et al. Deep learning enables reduced gadolinium dose for contrast-enhanced brain MRI. J Magn Reson Imaging 2018;48:330-40 CrossRef Medline

25. Therasse P, Arbuck SG, Eisenhauer EA, et al. New guidelines to evaluate the response to treatment in solid tumors: European Organization for Research and Treatment of Cancer, National Cancer Institute of the United States, National Cancer Institute of Canada. J Natl Cancer Inst 2000;92:205-16 CrossRef Medline

26. Wen PY, Macdonald DR, Reardon DA, et al. Updated response assessment criteria for high-grade gliomas: Response Assessment in Neuro-Oncology Working Group. J Clin Oncol 2010;28:1963-72 CrossRef Medline

27. Paek SH, Audu PB, Sperling MR, et al. Reevaluation of surgery for the treatment of brain metastases: review of 208 patients with single or multiple brain metastases treated at one institution with modern neurosurgical techniques. Neurosurgery 2005;56:1021-34; discussion 1021-34 Medline

28. Sahgal A, Ruschin M, Ma L, et al. Stereotactic radiosurgery alone for multiple brain metastases? A review of clinical and technical issues. Neuro Oncol 2017;19:ii2-15 CrossRef Medline

29. Kondziolka D, Kano H, Harrison GL, et al. Stereotactic radiosurgery 
as primary and salvage treatment for brain metastases from breast cancer: clinical article. J Neurosurg 2011;114:792-800 CrossRef Medline

30. Robson PM, Grant AK, Madhuranthakam AJ, et al. Comprehensive quantification of signal-to-noise ratio and g-factor for image-based and k-space-based parallel imaging reconstructions. Magn Reson Med 2008;60:895-907 CrossRef Medline

31. Sommer NN, Saam T, Coppenrath E, et al. Multiple sclerosis: improved detection of active cerebral lesions with 3-dimensional T1 black-blood magnetic resonance imaging compared with conventional 3-dimensional T1 GRE imaging. Invest Radiol 2018;53:13-19 CrossRef Medline

32. Rand S, Maravilla KR, Schmiedl U. Lesion enhancement in radiofrequency spoiled gradient-echo imaging: theory, experimental evaluation, and clinical implications. AJNR Am J Neuroradiol 1994; 15:27-35 Medline

33. Huber T, Alber G, Bette S, et al. Reliability of semi-automated segmentations in glioblastoma. Clin Neuroradiol 2017;27:153-61 CrossRef Medline

34. Huber T, Alber G, Bette S, et al. Progressive disease in glioblastoma: benefits and limitations of semi-automated volumetry. PLoS One 2017;12:e0173112 CrossRef Medline

35. McGraw KO, Wong SP. Forming inferences about some intraclass correlation coefficients. Psychol Methods 1996;1:30-46 CrossRef

36. Bland JM, Altman DG. Statistical methods for assessing agreement between two methods of clinical measurement. Lancet 1986;1: 307-10 Medline

37. Peat J, Barton B. Medical Statistics: A Guide to Data Analysis and Critical Appraisal. Oxford: Blackwell Publishing; 2005

38. Zach L, Guez D, Last D, et al. Delayed contrast extravasation MRI for depicting tumor and non-tumoral tissues in primary and metastatic brain tumors. PLoS One 2012;7:e52008 CrossRef Medline

39. Balériaux D, Colosimo C, Ruscalleda J, et al. Magnetic resonance imaging of metastatic disease to the brain with gadobenate dimeglumine. Neuroradiology 2002;44:191-203 CrossRef Medline

40. Cha S, Lupo JM, Chen MH, et al. Differentiation of glioblastoma multiforme and single brain metastasis by peak height and percentage of signal intensity recovery derived from dynamic susceptibility-weighted contrast-enhanced perfusion MR imaging. AJNR Am J Neuroradiol 2007;28:1078-84 CrossRef Medline

41. Krautmacher C, Willinek WA, Tschampa HJ, et al. Brain tumors full- and half-dose contrast-enhanced MR imaging at $3.0 \mathrm{~T}$ compared with 1.5 T-initial experience. Radiology 2005;237:1014-19 CrossRef Medline

42. Komada T, Naganawa S, Ogawa H, et al. Contrast-enhanced MR imaging of metastatic brain tumor at 3 Tesla: utility of $T(1)$ weighted SPACE compared with 2D spin echo and 3D gradient echo sequence. Magn Reson Med Sci 2008;7:13-21 CrossRef Medline

43. Reitsma JB, Rutjes AW, Khan KS, et al. A review of solutions for diagnostic accuracy studies with an imperfect or missing reference standard. J Clin Epidemiol 2009;62:797-806 CrossRef Medline

44. Sanai N, Berger MS. Surgical oncology for gliomas: the state of the art. Nat Rev Clin Oncol 2018;15:112-25 CrossRef Medline

45. Garcia MA, Lazar A, Duriseti S, et al. Discovery of additional brain metastases on the day of stereotactic radiosurgery: risk factors and outcomes. J Neurosurg 2017;126:1756-63 CrossRef Medline

46. Yang S, Nam Y, Kim MO, et al. Computer-aided detection of metastatic brain tumors using magnetic resonance black-blood imaging. Invest Radiol 2013;48:113-19 CrossRef Medline

47. Cui Y, Tha KK, Terasaka S, et al. Prognostic imaging biomarkers in glioblastoma: development and independent validation on the basis of multiregion and quantitative analysis of MR images. Radiology 2016;278:546-53 CrossRef Medline

48. Caulo M, Panara V, Tortora D, et al. Data-driven grading of brain gliomas: a multiparametric MR imaging study. Radiology 2014;272: 494-503 CrossRef Medline

49. Narang S, Lehrer M, Yang D, et al. Radiomics in glioblastoma: current status, challenges and potential opportunities. Transl Cancer Res 2016;5:383-97 CrossRef

50. Gillies RJ, Kinahan PE, Hricak H. Radiomics: images are more than pictures, they are data. Radiology 2016;278:563-77 CrossRef Medline 